\title{
Drift chamber calibration and particle identification in the P-349 experiment
}

D. Alfs ${ }^{1,2, *}$, A. Asaturyan ${ }^{3}$, M. Carmignotto ${ }^{4}$, M. Diermaier ${ }^{5}$, W. Eyrich ${ }^{6}$, B. Głowacz ${ }^{2}$, D. Grzonka ${ }^{1}$, F. Hauenstein ${ }^{7}$, T. Horn ${ }^{4}$, K. Kilian ${ }^{1}$, D. Lersch ${ }^{1}$, S. Malbrunot-Ettenauer ${ }^{8}$, A. Mkrtchyan ${ }^{3}$, H. Mkrtchyan ${ }^{3}$, P. Moskal $^{2}$, P. Nadel-Turonski ${ }^{9}$, W. Oelert ${ }^{10}$, J. Ritman ${ }^{1}$, T. Sefzick ${ }^{1}$, V. Tadevosyan ${ }^{3}$, E. Widmann ${ }^{5}$, M. Wolke ${ }^{11}$, S. Zhamkochyan ${ }^{3}$, M. Zieliński ${ }^{2}$, A. Zink ${ }^{12}$, and J. Zmeskal ${ }^{5}$

${ }^{1}$ Institut für Kernphysik, Forschungszentrum Jülich, Jülich, Germany

${ }^{2}$ M. Smoluchowski Institute of Physics, Jagiellonian University, Kraków, Poland

${ }^{3}$ A.I. Alikhanyan Science Laboratory, Yerevan, Armenia

${ }^{4}$ Physics Department, The Catholic University of America, Washington DC, USA

${ }^{5}$ Stefan-Meyer-Institut für subatomare Physik, Wien, Austria

${ }^{6}$ Universität Erlangen, Erlangen, Germany

${ }^{7}$ Old Dominion University, Norfolk, Virginia, USA

${ }^{8}$ Physics Department, CERN, Geneve, Switzerland

${ }^{9}$ Thomas Jefferson National Accelerator Facility, Newport News, Virginia, USA

${ }^{10}$ Johannes Gutenberg-Universität Mainz, Mainz, Germany

${ }^{11}$ Department of Physics and Astronomy, Uppsala University, Uppsala, Sweden

${ }^{12}$ Erlangen Centre for Astroparticle Physics (ECAP), Erlangen, Germany

\begin{abstract}
The goal of the P-349 experiment is to test whether $3.5 \mathrm{GeV} / \mathrm{c}$ antiprotons produced in high-energy proton-proton collisions are polarized in view of the preparation of a polarized antiproton beam. In this article, we present the details of the ongoing analysis focused on the drift chambers calibration and particle identification with DIRC.
\end{abstract}

\section{Introduction}

The experimental determination of the antiproton polarization in the P-349 experiment is done by the measurement of the left-right asymmetry of elastic antiproton scattering on a liquid hydrogen target in the Coulomb-nuclear interference region [1,2].

The experiment was performed in the CERN/PS test beam East Area. The secondary beam of positively charged particles with momentum equal to $3.5 \mathrm{GeV} / \mathrm{c}$ was delivered to the experimental setup in the form of $400 \mathrm{~ms}$ long spills. The experimental setup was designed to provide a precise tracking, online suppression of the dominant pionic background and reliable offline antiproton identification. For further information see $[1,3]$.

\footnotetext{
*e-mail: d.alfs@fz-juelich.de
} 


\section{Track reconstruction}

Tracking is done with a set of three drift chambers [4]: one drift chamber with 7 layers of hexagonally shaped cells (HEX, three layers with vertical wires, two double layers with wires inclined under $\pm 11^{\circ}$ ) placed in front of the scattering target and a package of two drift chambers with rectangular cells (D1, D2) for the scattered particles (in total 14 tracking layers: 3 double layers with straight wires and 2 double layers with wires inclined under $\pm 31^{\circ}$ ).

According to preliminary calculations, the maximum of the analyzing power $\mathrm{A}_{\mathrm{y}}$ equal to about $-4.5 \%$ is reached for a squared four-momentum transfer $|t| \cong 0.003(\mathrm{GeV} / \mathrm{c})^{2}[5]$ which corresponds to a scattering angle in the laboratory frame in the range of $10-20 \mathrm{mrad}$. Therefore, the required track reconstruction precision expected to be sufficient for the asymmetry determination is a few mrad.

In order to reach the desired precision, an iterative drift-time space calibration was performed for each wire layer separately. The resulting position resolution for a single layer is equal to 150 - $300 \mu \mathrm{m}$ [6]. In the next step the relative positioning of the drift chambers (including shifts and rotations) was corrected: first for one of the rectangular cell drift chambers with respect to the other, then (based on the sample of unscattered tracks) for the hexagonal cell drift chamber with respect to the rectangular cell drift chambers package.

In the position fine tuning procedure a set of events was chosen and track reconstruction was performed for different shifts (rotations) of the tuned chamber. Then the mean value of $\chi^{2} / \mathrm{NDF}$ (NDF - number of degrees of freedom) from the track fit was determined and the minimum of the $\chi^{2} / \mathrm{NDF}$ distribution was used as correction (see Fig. 1 left). This procedure was performed iteratively for the shifts (rotations) in all three directions (around all three axis) until the obtained values of position corrections reached zero within errors.

Track reconstruction accuracy was estimated using a sample of events for which a reconstruction of a single track through all drift chambers was possible (the choice was based on the $\chi^{2} / \mathrm{NDF}$ value). For these events which are considered as unscattered particles the tracks before and after the target passage were reconstructed separately and the angular difference between the projections of these tracks onto a horizontal plane was calculated (see Fig. 1 right for an example). The resulting track reconstruction accuracy is currently equal to about $5 \mathrm{mrad}$. This result still can be improved by performing a track angle dependent calibration for each drift cell separately (the dimensions of the drift chambers were big in comparison to the expected antiproton beam size) and fine tuning of the positioning.
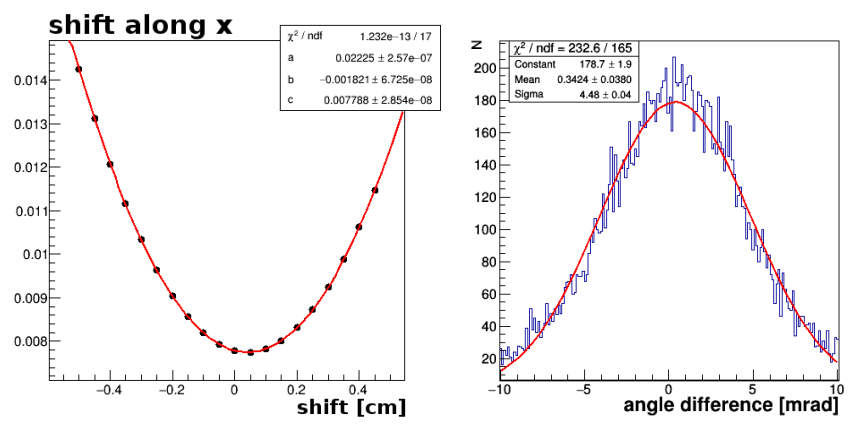

Figure 1. Left: Determination of the shift of the HEX drift chamber with respect to D1D2 drift chamber. Right: Estimation of the current track resolution (explanation given in the text). 


\section{Particle identification}

Particle identification is based on information from an aerogel Cherenkov detector and a Plexiglas DIRC detector. The former was used as an online trigger veto to reduce the pion background: the refractive index of the aerogel $(n=1.03)$ was chosen so that Cherenkov light is produced when a $3.5 \mathrm{GeV} / \mathrm{c}$ pion passes through it but no photons are created for an antiproton of the same momentum. In the Plexiglas DIRC all particles create Cherenkov light and the particle identification is done by an offline analysis of the Cherenkov cone angles. The detector geometry covering roughly a quarter of the full angle allows for detection of a part of photons from a Cherenkov ring (see Fig. 1 left).

The preliminary PID determined from the DIRC photon distributions for an event sample with the aerogel Cherenkov detector veto on and off is shown in the Fig. 2 middle and right, respectively, where separate maxima corresponding to the antiprotons and pions are visible. Reduction in the part corresponding to pions by rejection of events with a signal from the aerogel Cherenkov detector is significant, however, the dominant contribution is still due to pions (the threshold for the veto signal was adjusted to an online pion reduction by about a factor of 30).

Propagation of Cherenkov light and its reflections in the plexiglas and lightguides depend on the track angle and position. Therefore, complicated patterns can be created on the PMT matrices. A careful pion-antiproton separation requires a Monte Carlo supported analysis of the photon distribution which is currently being developed.
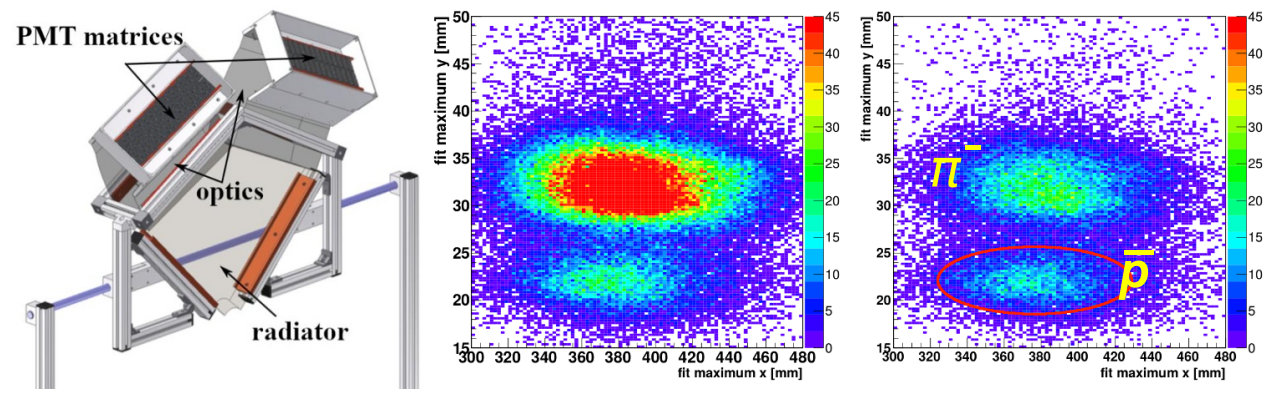

Figure 2. Left: Plexiglas DIRC geometry. Middle/Right: Preliminary separation of antiprotons and pions in the plexiglas DIRC without (middle) and with (right) a cut on a signal from aerogel Cherenkov detector.

\section{Summary and outlook}

The analysis of the existing data is ongoing with the goal of estimation of the number of available antiproton scattering events within the interesting kinematic range. The focus is now on optimization of the detector positioning with all particle tracks passing through the drift chambers and improvement of the drift-time space relations for the cells irradiated with antiprotons in order to reach the desired track reconstruction precision. In parallel, the DIRC response is investigated with MC generated photon distributions for a reliable particle identification.

Acknowledgements: This work was supported by Marie Skłodowska-Curie Innovative Training Network Fellowship of the European Commission's Horizon 2020 Programme (No. 721559 AVA), the 
Polish Ministry of Science and Higher Education and DAAD from resources of Bundesministerium für Bildung und Forschung (BMBF), by Marian Smoluchowski Kraków Research Consortium "Matter-Energy-Future (KNOW) and the Polish Ministry of Science and Higher Education through grant number 7150/E-338/M/2018.

\section{References}

[1] D. Grzonka et al., Acta Phys. Polon. B 46191 (2015)

[2] K. Kilian et al., Int. J. Mod. Phys. A 26, 757 (2011)

[3] D. Grzonka et al., contribution to these proceedings

[4] J. Smyrski et al., Nucl. Instrum. Meth. A 541, 574 (2005)

[5] J. Haidenbauer, private communication

[6] D. Alfs et al., Acta Phys. Polon. B 48, 1983 (2017) 